\title{
Cryo-HRSEM Techniques for Biological and Soft Materials Specimens
}

\author{
C. Frethem*
}

*University of Minnesota, Institute of Technology, Characterization Facility, 1-225 Nils Hasselmo Hall, Mail Stop 1191, 312 Church Street SE, Minneapolis, MN 55455

Modern scanning electron microscopes with field emission guns and cryo stages (Cryo-FESEMs) make it possible to achieve resolution in the macromolecular $(2-3 \mathrm{~nm})$ range on cryo-preserved biological (Fig.1) and soft materials specimens (Fig. 2) [1,2]. High resolution cryo-backscattered electron (BSE) imaging has been demonstrated in both in-lens and below-the-lens design cryoFESEMs [3]. For the purposes of this tutorial such instruments will be referred to as Cryo-High Resolution SEMs (Cryo-HRSEMs). Essential for successful use of Cryo-HRSEMs are methods developed to preserve nanoscale structures in their native hydrated state and render them visible with a high degree of fidelity while carefully avoiding the creation of artifacts during both sample preparation and observation $[1,2]$.

Specimen preparation for Cryo-HRSEM involves state-of-the-art techniques consisting of four major steps $[1,2]$ :

1) Cryo-Immobilization: Rapidly freezing the specimen to arrest physiological, chemical, and mechanical processes, to convert water to vitreous ice, and prevent the formation of crystalline ice which distorts or destroys the structure of the specimen.

2) Controlled Freeze-Drying: Removing vitreous ice by high-vacuum sublimation (etching) at -105 ${ }^{\circ} \mathrm{C}$ to $-95{ }^{\circ} \mathrm{C}$ to reveal embedded structures and increase topographic contrast. The bound water associated with molecular surfaces must be retained to prevent molecular collapse. In some samples surfaces to be etched must first be exposed by cryo-fracturing or cryosectioning / cryoplaning. 3) Cryo-Coating: Coating the specimen surface with a thin metal layer, such as $\mathrm{Cr}, \mathrm{Pt}$, W, or combinations such as $\mathrm{Pt} / \mathrm{C}$ followed by $\mathrm{C}$ in the double-layer coating technique [4] to optimize secondary and / or backscattered electron signal collection and to suppress charging and specimen damage due to electron beam irradiation.

4) Cryo-HRSEM Observation: Upon completion of the preparatory steps the sample must be maintained on a cryo stage within the sample chamber of the SEM. The choice of instruments and instrument operating parameters such as accelerating voltage, secondary (SE) vs backscattered (BSE) electron imaging, stage temperature control, etc., depends upon sample type, sample size, preparation methods, etc.

The tutorial will focus on recent past and current Cryo-HRSEM activity in our facility, along with a brief historical overview of the development of techniques, discussion of published methods beyond our facility's current capabilities, and future perspectives.

References

[1] R. Apkarian, Microsc. Microanal 9, 272, 2003

[2] S.L. Erlandsen et al., BioTechniques 31(2) (2001) 300-305

[3] S.L. Erlandsen et al., Microsc. Microanal. 9 (Suppl 2) (2003) 250-251

[4] P. Walther et al., Microsc. 179 (1995) 229-237 


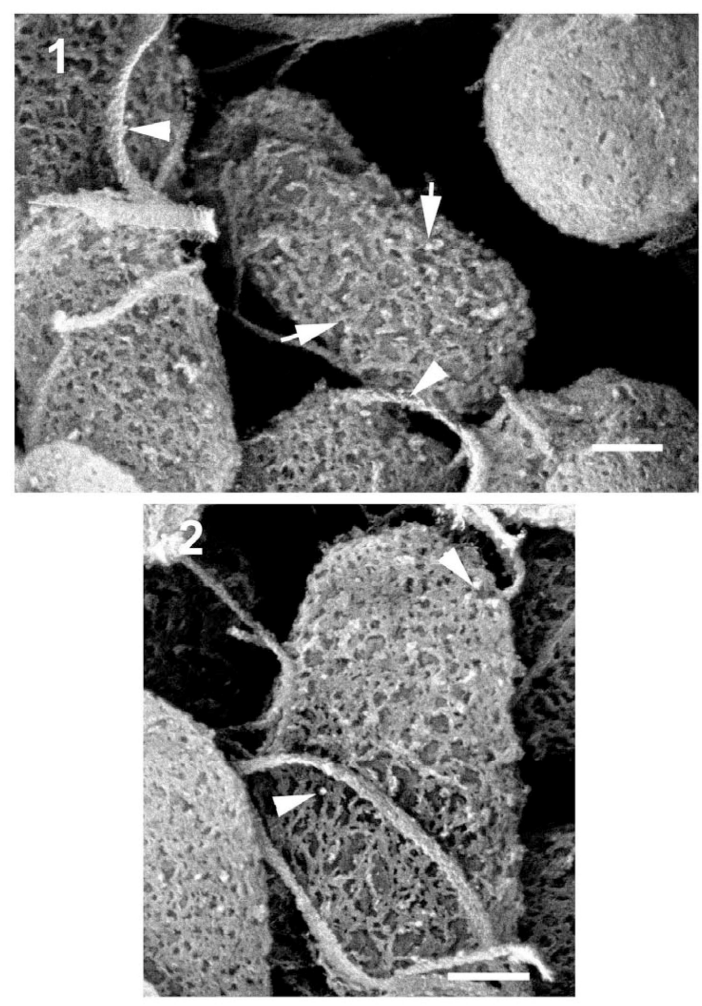

FIG. 1. (1). High resolution BSE cryoSEM of P. mirabilis taken in a below-the-lens FESEM. Observe network of capsular material covering cell and periodic pattern in the flagella (arrowheads). $10 \mathrm{~nm}$ colloidal gold (arrows), Magnification bar, $250 \mathrm{~nm}$.

(2). High resolution cryoSEM of P. mirabilis taken in an in-lens FESEM. Compare capsular material and flagellar structure with Figure $1.10 \mathrm{~nm}$ colloidal gold (arrows), magnification bar, $250 \mathrm{~nm}$. (Reproduced by permission from Erlandsen et al, Microsc. Microanal. 9 (Suppl 2), 2003.

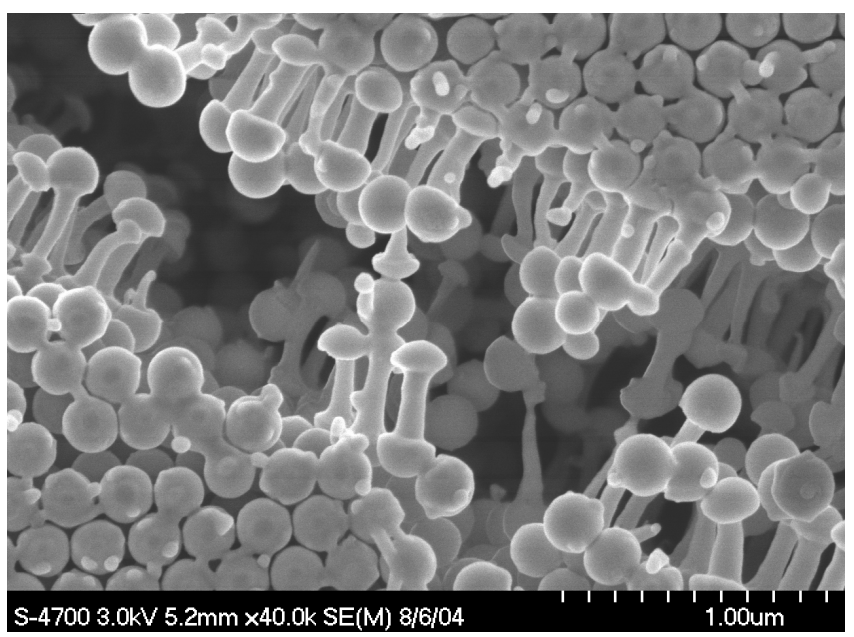

FIG. 2. A crack in a latex coating induced by plunge freezing into liquid ethane. Latex particles are elongated to form pull-outs, indicative of ductility even at $-183^{\circ} \mathrm{C}$. Reproduced by permission from Xiaobo Gong; University of Minnesota. 